\title{
Seclusion and the use of antipsychotics in hospitalized psychiatric patients
}

\author{
JOOST J. STOLKER ${ }^{1,2}$, GERARD W. K. HUGENHOLTZ ${ }^{1,2}$, EIBERT R. \\ HEERDINK $^{2}$, HENK L. I. NIJMAN ${ }^{3,4}$, HUBERT G. M. LEUFKENS ${ }^{2}, \&$ \\ WILLEM A. NOLEN ${ }^{5}$
}

\author{
${ }^{1}$ Altrecht, Institute for Mental Health Care, Den Dolder, The Netherlands, ${ }^{2}$ Department of \\ Pharmacoepidemiology and Pharmacotherapy, Utrecht Institute for Pharmaceutical Sciences (UIPS), \\ Utrecht, The Netherlands, ${ }^{3}$ Forensic Psychiatric Hospital, De Kijvelanden, Poortugaal, The \\ Netherlands, ${ }^{4}$ Faculty of Social Sciences, Radboud University, Nijmegen, The Netherlands, and \\ ${ }^{5}$ Department of Psychiatry, University Medical Center Groningen, Groningen, The Netherlands
}

\begin{abstract}
The purpose of this study is to measure the incidence of seclusion, identify possible determinants of seclusion and to gain insight into the use of antipsychotics before and after seclusion in acutely admitted patients. In The Netherlands, special treatment methods including seclusion and involuntary medication may only be applied in involuntarily hospitalized patients in cases of danger to the patient or others, with exclusion of emergencies. Data were retrospectively collected over the years 1997-1999 from a consecutive sample of 996 patients hospitalized on adult psychiatric admission wards. Young age, low Global Assessment of Functioning score, involuntary hospitalization and manic episode were significantly associated with seclusion, applied in $28.6 \%$ of the patients. The median time from admission to seclusion among psychotic patients who used antipsychotics during the first week was 7 days. In patients not using antipsychotics this was 2.5 days. In a substantial proportion of the psychotic patients antipsychotic treatment was initiated during or shortly after seclusion with a two times higher risk on antipsychotics in secluded patients compared with nonsecluded patients (relative risk: $2 ; 95 \%$ confidence interval: $1.2-3.4$ ). It was concluded that the use of antipsychotics is associated with a later application of seclusion. In a substantial proportion of the patients, treatment with antipsychotics was initiated during or shortly after seclusion.
\end{abstract}

Keywords: Psychiatric hospitals, mental disorders, social isolation, aggression, violence, antipsychotic agents

\section{Introduction}

In psychiatric inpatient care seclusion is one of the strategies to cope with disruptive and violent behaviour (Mason, 1993; Nijman, Merckelbach, Allertz, \& a Campo, 1997; Sailas \&

Correspondence: Joost J. Stolker, MD, PhD, Altrecht, Institute for Mental Health Care, Dolderseweg 164, 3734 BN Den Dolder, The Netherlands. E-mail: j.stolker@altrecht.nl 
Fenton, 2001). Compared with the relatively large number of studies on the characteristics of secluded patients, situational factors in relation to seclusion have hardly been studied (Nijman, 1999; Sailas \& Fenton, 2001). Remarkably, this is also the case for the relationship between psychotropics and the application of seclusion.

In The Netherlands, involuntary hospitalization does not mean that the patients can be treated pharmacologically against their will. Special treatment methods such as seclusion and involuntary medication may only be applied in involuntarily hospitalized patients in cases of danger to the patient or others, with exclusion of emergencies.

In the current study among hospitalized patients on acute admission wards, we sought to identify determinants of seclusion. Furthermore, the relationship between seclusion and the use of antipsychotics was investigated.

\section{Materials and methods}

\section{Patients and data collection}

Data were retrospectively collected over the years 1997-1999 from a consecutive sample of 996 patients of 16 years or older concerning their first complete hospitalization of 4 days or longer during this period on one of the participating admission wards. The admission wards with a total of 250 beds are part of three general psychiatric hospitals in the centre of The Netherlands, recently merged to one large institute for mental health care with 1200 beds and a total catchment area of 720000 persons. Patient characteristics, data on the use of seclusion and information on the use of antipsychotics were extracted from the hospital database containing patient data and an anonymously linked automated database of the pharmacy. The study protocol was approved by the Scientific Committee and the board of the hospitals with respect to the privacy aspects.

\section{Psychiatric diagnosis and antipsychotic treatment}

At admission (multiple) psychiatric diagnoses according to DSM-IV criteria (American Psychiatric Association, 1994) including clinical disorders, personality disorders and the Global Assessment of Functioning (GAF) scores were established by the ward psychiatrists.

Medication was coded according to the WHO Anatomical Therapeutic Chemical (ATC) coding system. Data on antipsychotics (ATC code $=$ N05A) were collected excluding droperidol, levomepromazine and promethazine (since in The Netherlands these drugs are mainly prescribed for sedation) and excluding lithium (since it is mainly used as a mood stabilizer).

\section{Data analysis}

In a nested case-control analysis, secluded patients were compared with non-secluded patients. Odds ratios (quantitative measure reported in case-control studies that describes the increase - or decrease - in risk associated with a specific risk factor) were calculated for patient-related factors possibly associated with seclusion including gender, age, (in)voluntary hospitalization, psychiatric diagnosis and GAF score. Adjustment for confounding was performed by an unconditional logistic regression analysis with seclusion as dependent variable and patient-related factors as independent variables. In this analysis, all seclusions were included. 
In a follow-up analysis, the duration of stay from admission to the first seclusion was calculated comparing patients with and without using antipsychotics in the first week of hospitalization prior to seclusion. Patients secluded immediately at the time of admission were excluded, because no data on medication use prior to seclusion were available for these patients. We calculated hazard ratios (quantitative measure reported in survival analyses that describes the increase - or decrease - in speed with which one group is likely to experience the event) by using Cox proportional hazards survival analysis (type of regression analysis using "time to event" as outcome). Relative risks (quantitative measure reported in cohort studies used to describe the increase - or decrease - in risk associated with a specific risk factor) on seclusion were calculated for patients who used antipsychotics in the first week of hospitalization prior to seclusion compared with patients not using antipsychotics. Relative risks on starting antipsychotics were also calculated for patients not using antipsychotics prior to seclusion and starting antipsychotics during or shortly after seclusion. All analyses were performed with the SPSS package and EGRET.

\section{Results}

The mean age of the population of 996 patients was 38.0 years (median: 37.0 years; range: 16-84 years). The number of male patients, 507 (50.9\%), was almost equal to the number of female patients. The mean duration of hospitalization was 57.2 days (median: 24.0 days; range: 4-711 days). Psychotic disorders were most prevalent: 398 of the 996 patients $(40.0 \%)$. A little more than one quarter of the patients (i.e. 285 of 996 patients or 28.6\%) had been secluded at least once during their hospitalization.

In Table I factors (possibly) associated with seclusion are listed. Young age was significantly associated with seclusion as was a lower GAF score between 35 and 55 and below 35 indicating major impairment in functioning. Involuntarily hospitalized patients were more likely to be secluded than voluntarily hospitalized patients with an odds ratio of 4.9 (95\% confidence interval: 3.5-6.9). Of the psychiatric diagnoses, only bipolar disorder (manic episode) was significantly associated with seclusion.

The median time from admission to seclusion among patients with psychotic disorders who used antipsychotics during the first week was 7 days (mean: 21.6 days; range 1-235 days). In patients not using antipsychotics this was 2.5 days (mean: 15.2 days; range 1-213 days). Figure 1 shows results of a Kaplan-Meier plot during the first month of hospitalization in this population. We found that antipsychotic use was significantly associated with a later application of seclusion with an adjusted (gender, age, GAF score) hazard ratio of 0.6 (95\% confidence interval: $0.3-1.0)$.

We found that among patients with psychotic disorders, $15.3 \%$ who used antipsychotics prior to seclusion during the first week of hospitalization were secluded compared to $20.5 \%$ of the psychotic patients not using antipsychotics during the first week. This corresponds to a relative risk of 0.7 (95\% confidence interval: $0.5-1.2)$.

In patients diagnosed as non-psychotic at admission, 19 out of $44(43.2 \%)$ started using antipsychotics during or directly after seclusion compared to 52 out of $288(18.1 \%)$ of nonsecluded patients. This corresponds with a relative risk of 2.4 (95\% confidence interval: 1.6-3.6). When looking specifically at patients with psychotic disorders, we also found that seclusion relatively often preceded the prescription of antipsychotics with a relative risk of 2.0 (95\% confidence interval: $1.2-3.4$ ). 
Table I. Factors associated with seclusion in hospitalized patients $(n=996)$. Crude odds $\operatorname{ratios}^{1}$ and adjusted ${ }^{2}$ odds ratios with $95 \%$ confidence interval $(95 \% \mathrm{CI}$ ) of secluded patients compared to non-secluded patients. Significant associations are given in bold.

\begin{tabular}{|c|c|c|c|c|}
\hline & $\begin{array}{c}\text { Secluded } \\
(n=285)(\%)\end{array}$ & $\begin{array}{l}\text { Not secluded } \\
(n=711)(\%)\end{array}$ & $\begin{array}{l}\text { Unadjusted odds } \\
\text { ratio }(95 \% \mathrm{CI})\end{array}$ & $\begin{array}{l}\text { Adjusted odds } \\
\text { ratio }(95 \% \mathrm{CI})\end{array}$ \\
\hline \multicolumn{5}{|l|}{ Gender } \\
\hline Female & $119(41.8)$ & $370(52.0)$ & 1.0 (reference) & 1.0 (reference) \\
\hline Male & $166(58.2)$ & $341(48.0)$ & $1.5(1.1-2.0)$ & $1.4(1.0-1.8)$ \\
\hline \multicolumn{5}{|l|}{ Age } \\
\hline $16-30$ & $103(36.1)$ & $170(23.9)$ & $2.7(1.7-4.1)$ & $2.2(1.4-3.5)$ \\
\hline $30-40$ & $89(31.2)$ & $202(28.4)$ & $1.9(1.2-3.0)$ & $1.6(1.0-2.6)$ \\
\hline $40-50$ & $59(20.7)$ & $190(26.7)$ & $1.4(0.8-2.2)$ & $1.0(0.6-1.7)$ \\
\hline$>50$ & $34(11.9)$ & $149(21.0)$ & 1.0 (reference) & 1.0 (reference) \\
\hline \multicolumn{5}{|l|}{ Global Assessment of Functioning ${ }^{3}$} \\
\hline$<35$ & $79(39.8)$ & $109(26.1)$ & $2.3(1.3-4.0)$ & $3.4(1.8-6.3)$ \\
\hline $35-55$ & $96(49.0)$ & $240(57.4)$ & $1.3(0.7-2.1)$ & $2.0(1.1-3.7)$ \\
\hline$\leq 55$ & $22(11.2)$ & $69(16.5)$ & 1.0 (reference) & 1.0 (reference) \\
\hline \multicolumn{5}{|l|}{ Type of hospitalization } \\
\hline Voluntary & $164(57.5)$ & $620(87.2)$ & 1.0 (reference) & 1.0 (reference) \\
\hline Involuntary & $121(42.5)$ & $91(12.8)$ & $5.0(3.6-6.9)$ & $4.9(3.5-6.9)$ \\
\hline \multicolumn{5}{|l|}{ Psychiatric diagnosis (DSM-IV) } \\
\hline Psychotic disorder & $127(46.7)$ & $271(39.8)$ & $1.3(1.0-1.7)$ & $1.2(0.9-1.7)$ \\
\hline Depressive disorder & $34(12.5)$ & $123(18.1)$ & $0.6(0.4-1.0)$ & $0.7(0.4-1.1)$ \\
\hline Anxiety disorder & $15(5.5)$ & $32(4.7)$ & $1.2(0.6-2.2)$ & $1.5(0.8-3.1)$ \\
\hline Bipolar disorder (manic episode) & $37(13.6)$ & $58(8.5)$ & $1.7(1.1-2.6)$ & $1.8(1.1-3.1)$ \\
\hline Personality disorder & $60(22.1)$ & $150(22.1)$ & $1.0(0.7-1.4)$ & $1.2(0.8-1.8)$ \\
\hline
\end{tabular}

${ }^{1}$ Odds ratio: quantitative measure reported in case-control studies that describes the increase (or decrease) in risk associated with a specific risk factor.

${ }^{2}$ Adjusted for gender, age, type of hospitalization, psychiatric diagnosis.

${ }^{3} n=615$ due to missing values.

${ }^{4} n=952$ due to missing values.

${ }^{5}$ According to DSM IV, multiple diagnoses could be established.

\section{Discussion}

The overall prevalence of seclusion found in the current study was high compared with other European countries (Salib, Ahmed, \& Cope, 1998; Kaltiala-Heino, Korkeila, Tuohimaki, Tuori, \& Lehtinen, 2000). More than a quarter (i.e. 28.6\%) of hospitalized psychiatric patients were secluded at least once during admission. This may be related to the Dutch legal situation: involuntary hospitalization does not mean that the patient has to accept the proposed medication treatment. Another important factor is a shortage of staff members, because of which intensive coaching of the patient is not an alternative. Young age, low GAF score and involuntary hospitalization were significantly associated with seclusion. It is likely that, in particular, agitation and aggression are serious problems in the group of severely ill involuntarily hospitalized young patients for whom seclusion is needed. We found a significant association between bipolar disorder (manic episode) and seclusion, but rather unexpectedly and for unknown reasons, no clear association between psychotic disorder and seclusion. This is in contrast with various other studies, in which it was found that patients with psychotic disorders show an increased seclusion rate (Angold, 1989; Fisher, 1994; Mason, 1993). 


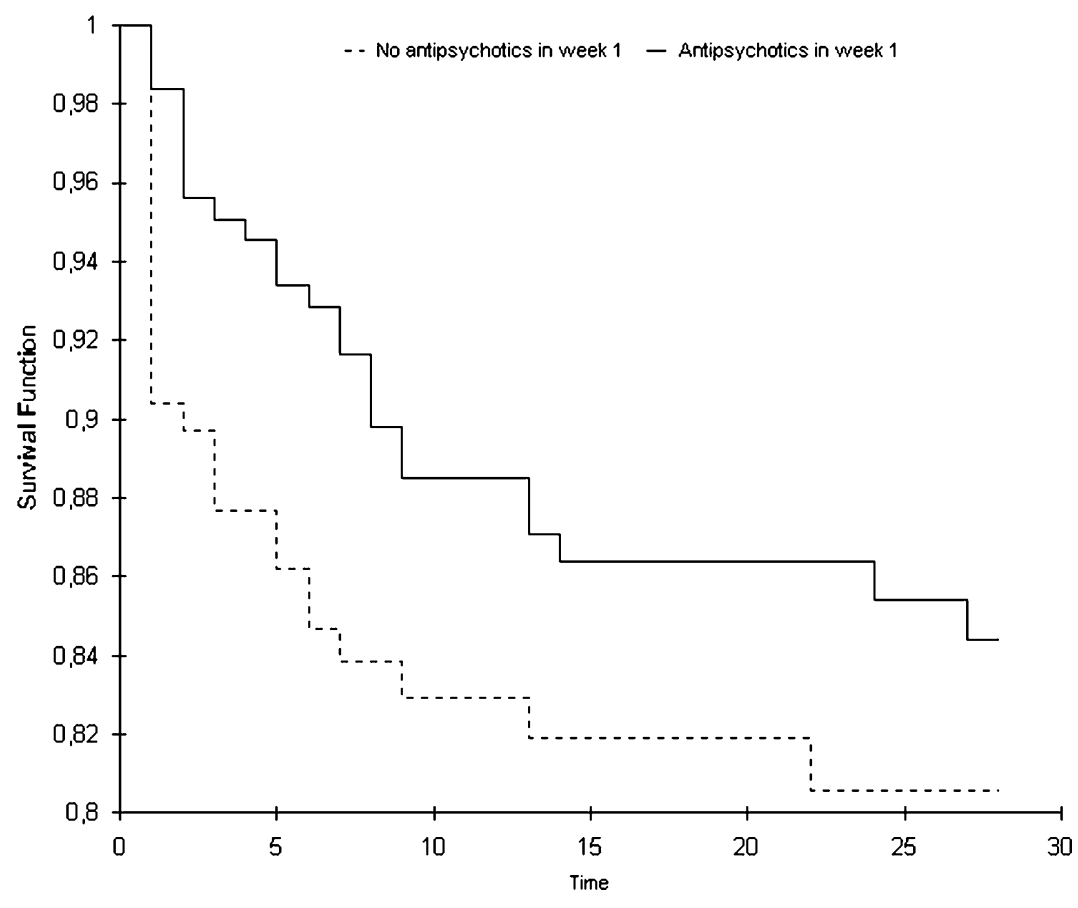

Figure 1. Survival analysis - Regression analysis using "time to seclusion" as outcome. Time from the first day of hospitalization to seclusion comparing patients using $(n=183)$ and not using antipsychotics $(n=146)$.

We found that the use of antipsychotics was significantly associated with a later application of seclusion. Patients with psychotic disorders who used antipsychotics during the first week of hospitalization were at a lower risk of seclusion than patients who did not use these agents. However, this difference was not statistically significant, probably due to small numbers of patients. Our findings are in line with the findings of Hoge et al. (1990) and Kasper, Hoge, Feucht-Haviar, Cortina, and Cohen (1997), who found that patients with psychotic disorders who refused treatment were significantly more likely to require seclusion. Our findings are also in line with the studies of Chiles, Davidson, and McBride (1994) and Chengappa et al. (2000) who found that treatment with either clozapine or risperidone was associated with a significant reduction in the use of seclusion. Apparently, in patients with psychotic disorders, not using antipsychotics is associated with aggression or violence for which seclusion is needed. Subsequently, in a substantial proportion of the patients antipsychotic treatment was initiated only during or directly after seclusion.

A limitation of our study is that $38.3 \%$ of the GAF scores at admission were missing in our database. Most of the missing scores were connected to the 1997 admissions when it was not yet common practice to record GAF scores into the hospital database compared to 1998 and 1999. However, we found that patients with available GAF scores did not differ in gender, age and only slightly in diagnostic categories when compared with patients with missing GAF scores. It is therefore likely that our data are representative for the total population of patients.

The associations found must be interpreted carefully concerning causality. Because of the observational study design, it is possible that the associations were influenced by confounding factors. The immediate reasons for the prescription of antipsychotics and the reasons why some psychotic patients did not use antipsychotics are not known. It is 
possible that patients not using antipsychotics prior to seclusion, probably due to noncompliance, were more severely ill and therefore more likely to be secluded than patients who used these agents (Hoge et al., 1990; Kasper, Hoge, Feucht-Haviar, Cortina, \& Cohen, 1997). However, after adjusting for GAF score we still found a significant hazard ratio.

To our knowledge, this is the first time that the relationship between antipsychotics and seclusion has been studied. According to Dutch law, involuntary hospitalization does not automatically mean that the patient can be involuntarily treated with psychotropics (this is in contrast with, for example, the $\mathrm{UK}$ ). The choice between coercive measures is left to the physician treating the patient. It should be primarily focused on the purpose of the measure, i.e. warding off serious danger, and it should infringe as little as possible on the rights of the patient. The results of our study suggest that seclusion is generally considered as less infringing than involuntary medication, but our results also suggest that in the end pharmacological treatment is inevitable anyway for a substantial proportion of secluded psychotic patients.

Our findings underline the question of what is to be preferred for patients who are aggressive or violent during their stay on an admission ward: seclusion or treatment with antipsychotics? Antipsychotics are considered essential in both international and the Dutch guidelines for the treatment of patients with psychotic disorders (American Psychiatric Association, 1997; Buitelaar et al., 1998). Moreover, antipsychotics are also indicated (and some are registered in The Netherlands) for the treatment of severe agitation including aggression. Seclusion may have serious physical and psychological adverse effects for the patient (Fisher, 1994; Lendemeijer \& Shortridge-Baggett, 1997). In addition, during the application of seclusion, staff members run the risk of getting injured (Carmel \& Hunter, 1989; Rice, Harris, Varney, \& Quincey, 1989). On the other hand, involuntary medication may also have considerable (psychological) adverse effects, which however has not been empirically substantiated (Hoge, 1998). No studies on the effectiveness of seclusion are available (Sailas \& Fenton, 2001).

In conclusion, we found that the use of antipsychotics was associated with a later application of seclusion, possibly a delay. Apparently, antipsychotics do lead to a reduction of agitation and aggression both in psychotic and non-psychotic patients. Thus, it is likely that their use also leads to a lower risk of seclusion. In a substantial proportion of our patients antipsychotic treatment was initiated shortly after starting seclusion. We think that earlier (involuntary) use of antipsychotics might have prevented patients from being secluded and we recommend more detailed investigations into the association between antipsychotics and seclusion.

\section{Acknowledgements}

The authors wish to thank R. H. Zuijderhoudt, MD, JD, for his valuable comments on an earlier version of this manuscript.

\section{References}

American Psychiatric Association (1994). Diagnostic and statistical manual of mental disorders (4th edn) (DSM-IV). Washington, DC: APA.

American Psychiatric Association (1997). Practical guideline for the treatment of patients with schizophrenia. Washington, DC: APA.

Angold, A. (1989). Seclusion. British fournal of Psychiatry, 154, 437-444. 
Buitelaar, J. K., van Ewijk, W. M., Harms, H. H., Kahn, R. S., Linszen, D. H., Loonen, A. J. M., et al. (1998). Richtlijn antipsychoticagebruik bij schizofrene psychosen. [Guideline antipsychotic treatment in schizophrenic psychosis]. Amsterdam: Nederlandse Vereniging voor Psychiatrie.

Carmel, H., \& Hunter, M. (1989). Staff injuries from inpatient violence. Hospital and Community Psychiatry, 40, 41-46.

Chengappa, K. N., Levine, J., Ulrich, R., Parepally, H., Brar, J. S., Atzert, R., et al. (2000). Impact of risperidone on seclusion and restraint at a state psychiatric hospital. Canadian fournal of Psychiatry, 45, 827-832.

Chiles, J. A., Davidson, P., \& McBride, D. (1994). Effects of clozapine on use of seclusion and restraint at a state hospital. Hospital and Community Psychiatry, 45, 269-271.

Fisher, W. A. (1994). Restraint and seclusion: A review of the literature. American fournal of Psychiatry, 151, $1584-1591$.

Hoge, S. K. (1998). Consequences of involuntary treatment (reply). American fournal of Psychiatry, $155,451$.

Hoge, S. K., Appelbaum, P. S., Lawlor, T., Beck, J. C., Litman, R., Greer, A., et al. (1990). Prospective, multicenter study of patients' refusal of antipsychotic medication. Archives of General Psychiatry, 47, 949-956.

Kaltiala-Heino, R., Korkeila, J., Tuohimaki, C., Tuori, T., \& Lehtinen, V. (2000). Coercion and restrictions in psychiatric inpatient treatment. European Psychiatry, 15, 213-219.

Kasper, J. A., Hoge, S. K., Feucht-Haviar, T., Cortina, J., \& Cohen, B. (1997). Prospective study of patients' refusal of antipsychotic medication under a physician discretion review procedure. American fournal of Psychiatry, 154, 483-489.

Lendemeijer, B., \& Shortridge-Baggett, L. (1997). The use of seclusion in psychiatry: a literature review. Scholarly Inquiry for Nursing Practice, 11, 299-315.

Mason, T. (1993). Seclusion theory reviewed - a benevolent or malevolent intervention? Medicine, Science and the Law, 33, 95-102.

Nijman, H. (1999). Aggressive behavior of psychiatric inpatients. Maastricht: Datawyse/Universitaire Pers Maastricht.

Nijman, H., Merckelbach, H. L., Allertz, W. F., \& a Campo, J. M. (1997). Prevention of aggressive incidents on a closed psychiatric ward. Psychiatric Services, 48, 694-698.

Rice, M. E., Harris, G. T., Varney, G. W., \& Quincey, V. L. (1989). Violence in institutions. Understanding, prevention and control. Toronto: Hogrefe \& Huber.

Sailas, E., \& Fenton, M. (2001). Seclusion and restraint for people with serious mental illnesses (Cochrane Review). In The Cochrane Library, 3, Oxford: Update Software.

Salib, E., Ahmed, A. G., \& Cope, M. (1998). Practice of seclusion: a five-year retrospective review in North Cheshire. Medicine, Science and the Law, 38, 321-327. 